\title{
Responsabilidad social en el gobierno y gestión de las universidades estatales chilenas $^{1}$
}

\author{
Social responsibility in the governance and management of \\ the chilean state universities \\ Recibido: 05 de noviembre de 2014 - Revisado: 27 de mayo de 2015 - Aceptado: 22 de septiembre de 2015
}

\author{
Ricardo Gaete Quezada²
}

\section{Resumen}

Se estudia la presencia de la responsabilidad social en planes estratégicos, organigramas y modelos educativos como ámbitos de gobierno y gestión de las universidades estatales chilenas, utilizando un enfoque de investigación cuantitativo -análisis de contenido-para determinar su frecuencia de aparición. Los resultados muestran una baja participación en la misión institucional y levemente superior en el resto de aspectos del gobierno y gestión universitaria analizados. Lo anterior, no implica un actuar socialmente irresponsable de las universidades de la muestra, pero sí puede transformarse en una barrera importante para avanzar en la incorporación de la responsabilidad social en la cultura y quehacer institucional en el largo plazo, como un elemento de la política pública de educación superior en Chile.

\section{Palabras clave}

Responsabilidad social universitaria, gobierno y gestión universitaria, universidades estatales chilenas, educación superior.

\begin{abstract}
The presence of social responsibility in strategic planning, organigrams and educational models as areas of governance and management of Chile's state universities are studied, using a quantitative approach - analysis of contentto determine their frequency of occurrence. The results show low participation in institutional mission and slightly higher in other analyzed aspects of government and university management. The above does not imply socially irresponsible acting of the universities in the sample, but it can become a major barrier to progress in incorporating social responsibility into the culture and institutional work in the long run, as an element of political public higher education in Chile.
\end{abstract}

\section{Keywords}

University social responsibility, university governance and management, Chilean state universities, higher education.

\footnotetext{
${ }^{1}$ El artículo es resultado del proyecto del Fondo Nacional de Desarrollo Científico y Tecnológico de Iniciación a la Investigación titulado "Una mirada a la responsabilidad social universitaria en la ciudad de Antofagasta" realizado en el 2012 y financiado por la Comisión Nacional de Ciencia y Tecnología de Chile.

2 Doctor en Procesos de Cambio Social por la Universidad de Valladolid, Valladolid, España. Docente del Departamento de Ciencias Sociales e investigador sobre la responsabilidad social universitaria, Universidad de Antofagasta, Antofagasta, Chile. Correo electrónico: ricardo.gaete@uantof.cl Para citar este artículo use: Gaete, R. (2015). Responsabilidad social en el gobierno y gestión de las universidades estatales chilenas. Revista Civilizar Ciencias Sociales y Humanas, 15(29), 163-180.
} 


\section{Introducción}

La relevancia de la responsabilidad social universitaria (RSU) es un hecho creciente en el mundo, que se ha consagrado especialmente a partir de los esfuerzos en el ámbito global de instituciones tales como la Organización de las Naciones Unidas para la Educación, la Ciencia y la Cultura (Unesco, por su sigla en inglés) o los desarrollados por el proyecto Tuning en América Latina. Además, en algunos países, distintos grupos de universidades han tomado la iniciativa en la implantación del comportamiento socialmente responsable como un aspecto importante en sus proyectos institucionales, como el caso de las universidades andaluzas en España, del proyecto Universidad Construye País (UCP) en Chile, del Observatorio de Responsabilidad Social Universitaria (ORSU) en Colombia, o incluso redes conformadas por universidades de diferentes naciones como la Asociación de Universidades confiadas a la Compañía de Jesús en América Latina (AUSJAL).

El creciente interés por la RSU debiera materializarse en algunas cuestiones específicas de las organizaciones universitarias e incidir en espacios concretos de su quehacer, uno de los cuales se trata con especial interés en este trabajo referido al gobierno y gestión universitaria.

En el artículo se examina el caso de las universidades estatales chilenas respecto de la consideración de la responsabilidad social como un factor clave en los principales aspectos de gobierno y gestión universitaria: los planes estratégicos, los modelos educativos y la estructura organizacional. Se realiza un análisis de contenido de los tres elementos para observar si aparece el término "responsabilidad social".

Los resultados obtenidos muestran una baja presencia de la RSU en la declaración de misión de las universidades, y un leve aumento de esta en la visión y en los valores institucionales, sin llegar a ser mayoritaria entre las instituciones analizadas. Esta situación se repite para el caso de la estructura organizacional y modelos educativos, con la excepción de la Universidad de La Frontera, quien es la única institución en estudio que considera al comportamiento socialmente responsable en todos los elementos examinados aquí.

La principal conclusión de la investigación ubica a la RSU como poco significativa dentro de los aspectos del gobierno y la gestión universitaria de las universidades estatales chilenas, sobre todo si se observa que no existe gran coherencia entre los planes estratégicos, los modelos educativos y la estructura organizacional en aquellas instituciones que usan dicho término.

\section{Marco de referencia}

En 1998, durante la celebración de la Conferencia Mundial sobre la Educación Superior realizada en París, la Unesco hizo un llamado a las universidades para que asumieran con mayor decisión su responsabilidad social, en particular en lo que se relaciona con la generación y transferencia del conocimiento, lo cual estableció uno de los antecedentes para el análisis de dicho concepto en el ámbito universitario.

En Chile, la RSU alcanzó un importante crecimiento y presencia entre las universidades chilenas a través del proyecto UCP, desarrollado entre los años 2001 y 2008 por la Fundación Avina y la Corporación Participa. En esta iniciativa participaron un total de catorce universidades chilenas que poco a poco se fueron integrando al proyecto, destacándose la heterogeneidad de las instituciones: universidades estatales y privadas, regionales y de la zona metropolitana, tradicionales pertenecientes al Consejo de Rectores de las Universidades Chilenas (CRUCH) y privadas de más reciente creación.

En la actualidad, el proyecto UCP ya no está en funcionamiento, no obstante algunas 
universidades continúan ejecutando iniciativas de RSU, en especial en lo relativo a la formación de profesionales socialmente responsables. Un ejemplo de ello, es el caso de la elaboración de un modelo educativo para la enseñanza de la responsabilidad social que se adelantó con financiamiento del Programa de Mejoramiento de la Calidad y la Equidad de la Educación Superior (MECESUP), del que forman parte cuatro universidades: Universidad de Concepción, Universidad de La Frontera, Pontificia Universidad Católica de Valparaíso y Universidad de Talca.

Así mismo, en la literatura es posible identificar diferentes propuestas conceptuales para incorporar a la RSU en el funcionamiento de las universidades, lo que según Gaete (2011) puede clasificarse en tres grandes enfoques, como se observa en la tabla 1.

Tabla 1

Diferentes enfoques del concepto de responsabilidad social universitaria en la literatura

\begin{tabular}{|c|c|}
\hline Enfoque & Concepto de RSU \\
\hline $\begin{array}{c}\text { Gerencial } \\
\text { (orientado a la transparencia y la } \\
\text { rendición de cuentas) }\end{array}$ & $\begin{array}{l}\text { Una política de mejora continua de la universidad hacia el cumplimiento efectivo de su misión } \\
\text { social mediante cuatro procesos: gestión ética y ambiental de la institución; formación de ciuda- } \\
\text { danos responsables y solidarios; producción y difusión de conocimientos socialmente pertinentes; } \\
\text { y participación social en promoción de un desarrollo más humano y sostenible (Vallaeys, 2008, } \\
\text { p. 209) }\end{array}$ \\
\hline $\begin{array}{l}\text { Transformacional (relacionado } \\
\text { con la asunción de un liderazgo } \\
\text { más activo de la universidad en } \\
\text { la sociedad) }\end{array}$ & $\begin{array}{l}\text { La habilidad y efectividad de la universidad para responder a las necesidades de transformación } \\
\text { de la sociedad donde está inmersa, mediante el ejercicio de sus funciones sustantivas: docencia, } \\
\text { investigación, extensión y gestión interna. Estas funciones deben estar animadas por la búsqueda } \\
\text { de la promoción de la justicia, la solidaridad y la equidad social, mediante la construcción de } \\
\text { respuestas exitosas para atender los retos que implica promover el desarrollo humano sustentable } \\
\text { (AUSJAL, 2009, p. 9) }\end{array}$ \\
\hline $\begin{array}{c}\text { Normativo } \\
\text { (afín con un intercambio de } \\
\text { valores universitarios y sociales) }\end{array}$ & $\begin{array}{l}\text { La capacidad que tiene la universidad como institución, de difundir y poner en práctica un con- } \\
\text { junto de principios y valores generales y específicos por medio de los procesos clave de gestión, } \\
\text { docencia, investigación y extensión, respondiendo socialmente así ante la comunidad universita- } \\
\text { ria y el país en que está inserta (Jiménez, 2002, p. 11) }\end{array}$ \\
\hline
\end{tabular}

Fuente: elaboración propia.

En la tabla 1 se evidencia la existencia de diferentes ámbitos o líneas de investigación que se vinculan con el concepto de RSU, destacando aquellos estudios que recogen los puntos de vista sobre el comportamiento socialmente responsable en las universidades desde la perspectiva de los alumnos (Arango, Clavijo, Puerta, \& Sánchez, 2014), los profesores universitarios (Ojeda, 2013) o los directivos universitarios (Aristimuño \& Rodríguez, 2014; Gaete, 2015). En confirmación de lo anterior, la Fundación Universitaria Luis Amigó (2015) editó un libro colectivo con diferentes experiencias iberoamericanas en RSU abarcando aspectos conceptuales, investigaciones o propuestas de instrumentos y modelos sobre dicho concepto.
En lo relativo a la aplicación de la RSU en el gobierno y la gestión universitaria, Larrán y Andrades (2015) en su análisis de distintos enfoques teóricos empleados para justificar el compromiso de las universidades con la responsabilidad social, señalan que desde la teoría de la agencia se plantea la necesidad de profesionalizar la gestión universitaria para fortalecer la autonomía y rendición de cuentas de las universidades, en particular para implantar mecanismos que mejoren la transparencia informativa de los impactos económicos, sociales y medioambientales del quehacer universitario. La propuesta de estos autores es coincidente con el enfoque gerencial de la RSU (véase tabla 1). 
Por su parte, Gaete (2012) hace una propuesta para fortalecer el gobierno universitario pluralista teniendo en cuenta la teoría de los stakeholders, para que estos aumenten la participación de diferentes actores sociales o partes interesadas en la toma de decisiones y evaluación de las diferentes funciones universitarias.

\section{El gobierno universitario moderno.}

Según la Comisión Europea (2008) es posible distinguir entre gobierno y gestión, donde el primero "hace hincapié en el proceso por el cual se establecen las políticas y objetivos a largo plazo, así como las estrategias para lograrlos", mientras que la segunda "se refiere a la puesta en marcha del conjunto de objetivos que persigue una institución de educación superior basándose en las normas establecidas" (p. 12).

De acuerdo con Castro y Tomás (2010a) los órganos del gobierno universitario se catalogan según su composición en unipersonales o colegiados y, según su ámbito de influencia, en generales o periféricos. No obstante, sin importar su clasificación, Ganga (2005) afirma que "las universidades al igual que cualquier tipo de organización necesitan de un sistema de gobierno que posibilite la planificación, organización, dirección y control de su quehacer inmediato y de largo plazo" (p. 222).

La literatura tradicional sobre el gobierno universitario con una fuerte influencia weberiana propone tres modelos: burocrático, colegial y político. Sin embargo, en las últimas décadas el ingreso del sector privado al escenario universitario, ha permitido el desarrollo de un modelo de gobierno universitario adicional: el de mercado (Brunner, 2011; Castro \& Tomás, 2010b). Estos modelos se describen a continuación:

Colegial: viene de la expresión de la tradición e ideología originarias de las universidades en la Edad Media en donde, las decisiones se toman en régimen de autogobierno por parte de la universidad, orientadas por el criterio de su personal académico, sin mayores condicionamientos pues se reconoce la autonomía del alma mater para gobernarse y decidir su propio funcionamiento.

Burocrático: se relaciona con los modelos alemán y francés de universidad. En él las decisiones institucionales están condicionadas por alguna instancia de la administración pública.

Político: es caracterizado por una fuerte competencia por el control del poder en el interior de las universidades, donde grupos con diferentes intereses y valores se disputan la tuición de los procesos y resultados organizacionales, haciendo necesario que las autoridades universitarias desarrollen un permanente proceso de negociación y mediación.

De mercado: en él las decisiones se orientan a la venta de servicios de enseñanza, asesoramiento e investigación, pues el Estado no financia directamente a la universidad sino que facilita los fondos a los estudiantes a través de becas y préstamos, para que sean ellos quienes se dirijan al proveedor de los servicios universitarios.

Además, Brunner (2011) plantea dos modelos de gobierno y gestión universitaria que profundizan el gobierno de mercado:

De partes interesadas: combina emprendimiento con colegialidad, ofreciendo una visión más amplia y compleja de las funciones y responsabilidades universitarias que desde este modelo se preocupan por los intereses de los stakeholders internos (profesores, funcionarios, estudiantes) y externos (e. g. municipios, empleadores, titulados y ONG), mostrando una mayor apertura hacia la sociedad civil y demás actores sociales.

Emprendedor: las prioridades y toma de decisiones se dirigen hacia la obtención de recursos y reputación. Está caracterizado por un 
liderazgo que se orienta al cambio, una columna vertebral de departamentos, escuelas y facultades con ejecutivos intermedios capaces de implementar las decisiones del principal, una red que vincula a la universidad con su entorno, una base diversificada de ingresos y por el desarrollo de una cultura emprendedora institucional.

Así, Brunner (2011) sitúa su propuesta dentro de las tendencias evolutivas de la gobernanza universitaria, definiendo dicho concepto como

[...] la manera en que las instituciones se hallan organizadas y son operadas internamente -desde el punto de vista de su gobierno y gestión- $\mathrm{y}$ sus relaciones con entidades y actores externos con vistas a asegurar los objetivos de la educación superior (p. 137).

E identificando claramente dónde se deben desplegar los esfuerzos relativos al gobierno y la gestión universitaria. De esta forma, la relación de la universidad con sus partes interesadas se transforma en un aspecto central de la manifestación de la responsabilidad social en el gobierno y gestión universitaria, tanto respecto de la identificación de aquellas sobre las cuales las universidades deben incorporar a sus procesos decisionales (Gaete, 2011), como respecto del diálogo y respuestas a sus necesidades y demandas (Jongbloed \& Goedegebuure, 2003).

\section{Aspectos destacados en la gestión universitaria.}

Antes de analizar algunos aspectos relevantes del concepto de gestión universitaria, se destaca la reflexión de Meléndez, Solís y Gómez (2010) en cuanto a la paradoja existente en la literatura y que se ha concentrado en esclarecer quién o quiénes deben tener el poder de tomar las decisiones pero soslayando el examen específico de los procesos decisorios y sus efectos proyectados o imprevistos, es decir, una literatura más preocupada de las estructuras de gobierno que de la gestión universitaria.
Según Lopera (2004), la gestión universitaria incluye "el conjunto de estrategias dirigidas por personas y cuerpos colegiados, de mando directivo, para garantizar el cumplimiento del proyecto educativo bajo condiciones sostenibles y viables institucionalmente" (p. 618), e implica que además de las habilidades técnicas para tomar decisiones oportunas, un directivo universitario debe ejecutar estrategias políticas y culturales para mantener un adecuado nivel de gobernabilidad de la universidad, de forma que sea capaz de: atender de manera apropiada las demandas internas mediante el correcto uso del poder y autoridad de los directivos universitarios, resolver conflictos y generar acuerdos internos necesarios para asegurar la estabilidad institucional. Esto, puede verse fuertemente condicionado por la existencia de un modelo de gobierno colegial o político, según las tipologías que se comentaron en el apartado anterior.

A la luz de estos antecedentes, resulta conveniente citar las palabras de Rodríguez (2006) en el contexto de su análisis sobre las decisiones estratégicas de las universidades públicas chilenas, que destacan la gran relevancia de la gestión universitaria para este tipo de instituciones:

Operan en un mismo sector industrial, $[\ldots]$ tienen una constitución jurídica y desafíos equivalentes, entre otras similitudes, pueden y suelen tener distintos resultados. De hecho algunas son más exitosas que otras. Estas diferencias se explican de manera significativa por la calidad de las decisiones estratégicas adoptadas (p. 50).

Así mismo, según Atria (2006) la experiencia de los procesos de acreditación institucional de las universidades chilenas del Consejo de Rectores ha permitido identificar tres temas transversales, fundamentales para la buena gestión institucional de este tipo de universidades: a) cautela o resguardo de la misión institucional; b) procesos de planificación estratégica; y c) estructura orgánica para la gestión. 
De esta manera, el gobierno universitario nos aporta la perspectiva más estructural que se asocia con la determinación de "cuáles" son las instancias unipersonales o colegiadas que tienen la responsabilidad de tomar decisiones dentro de una universidad, habitualmente reflejadas en los organigramas de las universidades. En cambio, la gestión universitaria nos proporciona la perspectiva procedimental que debiera preocuparse por los impactos del quehacer universitario, haciendo énfasis en el "cómo" se implementan las decisiones y alcanzan los resultados organizacionales, lo que para nuestro estudio de caso se verá en el análisis de los planes estratégicos y modelos educativos.

\section{Metodología}

Considerando un enfoque o paradigma de investigación cuantitativo, el presente estudio de carácter exploratorio-descriptivo examina el uso de la expresión "responsabilidad social universitaria" en los planes estratégicos, organigramas y modelos educativos de las universidades estatales chilenas.

\section{Del universo y la muestra del estudio.}

El universo que se tiene en cuenta para el presente estudio son las sesenta universidades que actualmente integran el sistema universitario chileno, que se distribuyen en veinticinco universidades tradicionales que integran el CRUCH $\mathrm{y}$ treinta y cinco universidades privadas.

La muestra del estudio es no probabilística de tipo intencional debido a que las instituciones seleccionadas representan casos típicos. Está integrada por las dieciséis universidades estatales (doce regionales y cuatro metropolitanas) que integran el CRUCH, lo que equivale al $27 \%$ del universo. Dicha instancia colegiada además está compuesta por otras nueve universidades tradicionales privadas con aporte (tres laicas y seis confesionales católicas) que completan el total de veinticinco universidades del CRUCH, pero que difieren en cuanto a sus características respecto de las universidades estatales, por lo que la muestra es intencional hacia este último tipo de universidades por su mayor homogeneidad. Algunas estadísticas básicas de las instituciones universitarias que comprenden la muestra del presente estudio se presentan en la tabla 2.

Tabla 2

Estadísticas universidades estatales chilenas

\begin{tabular}{|c|c|c|c|c|c|c|}
\hline \multirow{2}{*}{ Universidad } & \multirow{2}{*}{$\begin{array}{l}\text { Acreditación } \\
\text { institucional }\end{array}$} & \multirow{2}{*}{$\begin{array}{c}\text { Alumnos } \\
\text { pregrado } \\
2015\end{array}$} & \multirow{2}{*}{$\begin{array}{l}\text { Promedio } \\
\text { PSU }^{1} \\
2015\end{array}$} & \multicolumn{3}{|c|}{ Establecimiento de origen alumnos pregrado 2015 (\%) } \\
\hline & & & & Municipal & Subvencionado & Particular \\
\hline Arturo Prat & Tres años (2017) & 13.329 & 529 & 39,3 & 59,2 & 1,5 \\
\hline de Antofagasta & Cuatro años (2017) & 7.427 & 538 & 36,7 & 50,3 & 13,0 \\
\hline de Atacama & Cuatro años (2017) & 4.352 & 544 & 40,5 & 56,6 & 2,9 \\
\hline de Chile & Siete años (2018) & 29.494 & 667 & 27,7 & 37,5 & 34,8 \\
\hline de La Frontera & Cinco años (2018) & 9.388 & 591 & 31,7 & 62,2 & 6,1 \\
\hline de La Serena & Cuatro años (2016) & 6.997 & 574 & 22,3 & 74,3 & 3,4 \\
\hline de Los Lagos & Tres años (2016) & 9.238 & 534 & 53,9 & 44,7 & 1,4 \\
\hline de Magallanes & Cuatro años (2019) & 4.243 & 535 & 40,8 & 52,0 & 7,2 \\
\hline de Playa Ancha & Cuatro años (2016) & 8.209 & 551 & 27,0 & 68,5 & 4,5 \\
\hline de Santiago de Chile & Seis años (2020) & 21.988 & 605 & 28,2 & 62,4 & 9,4 \\
\hline de Talca & Cinco años (2019) & 9.392 & 595 & 40,2 & 51,1 & 8,7 \\
\hline de Tarapacá & Cinco años (2017) & 8.722 & 533 & 37,1 & 59,2 & 3,7 \\
\hline de Valparaíso & Cinco años (2017) & 14.336 & 586 & 19,9 & 65,3 & 14,8 \\
\hline del Bío-Bío & Cinco años (2019) & 11.956 & 563 & 33,0 & 63,7 & 3,3 \\
\hline Metropolitana de Ciencias de la Educación & Cuatro años (2016) & 4.700 & 595 & 30,8 & 61,3 & 7,9 \\
\hline Tecnológica Metropolitana & Tres años (2016) & 7.613 & 559 & 28,6 & 67,6 & 3,8 \\
\hline
\end{tabular}

Fuente: elaboración propia con base en el portal web de Mifuturo.cl 
Paralelamente al CRUCH, las 16 universidades estatales chilenas se encuentran subagrupadas en el Consorcio de Universidades del Estado de Chile y como se observa en la tabla 2 , el $44 \%$ de ellas posee cinco o más años de acreditación, con una matrícula total para el año 2015 de 171.384 estudiantes de pregrado; solamente dos universidades (de Chile y de Santiago de Chile) superaron los 600 puntos promedio en la Prueba de Selección Universitaria (PSU) el año 2015 y tienen el mayor número de alumnos matriculados ese año; solo cuatro universidades estatales (Atacama, de Los Lagos, de Magallanes y de Talca) matricularon a más del $40 \%$ de sus estudiantes desde establecimientos secundarios de la educación pública municipal, existiendo una correlación directa con aquellas universidades que matricularon alumnos con promedio PSU más bajo de la muestra (Arturo Prat, de Antofagasta de Los Lagos, de Magallanes y de Tarapacá) con menos de 540 puntos, todas universidades pertenecientes a las zonas extremas del país.

A partir del perfil de la muestra analizada, surgen algunas preguntas de investigación que orientan este estudio: ¿cuál es el grado de pre- sencia y relevancia de la RSU en el gobierno y la gestión de las universidades estatales chilenas? ¿Existe más alta participación de la responsabilidad social en el gobierno y la gestión en aquellas universidades estatales que matriculan una mayor cantidad de alumnos que provienen de establecimientos municipales? ¿Hay mayor presencia de la responsabilidad social en el gobierno y la gestión universitaria en aquellas instituciones con más tiempo de acreditación?

\section{Objetivo del estudio y técnica de recolección de datos.}

El objetivo del estudio es examinar la envergadura y presencia de la RSU en el gobierno y gestión de las universidades estatales chilenas, para lo cual se desarrolla un análisis de contenido de diferentes aspectos relacionados con el gobierno y la gestión de dichas organizaciones universitarias (planes estratégicos, organigramas y modelos educativos). Según Palomares, García y Castro (2012) este procedimiento de análisis requiere desplegarse a través de unidades de análisis, las que se estructuran en unidades de muestreo, contexto y registro, como se observa en la tabla 3.

Tabla 3

Estructura del análisis de contenido desarrollado

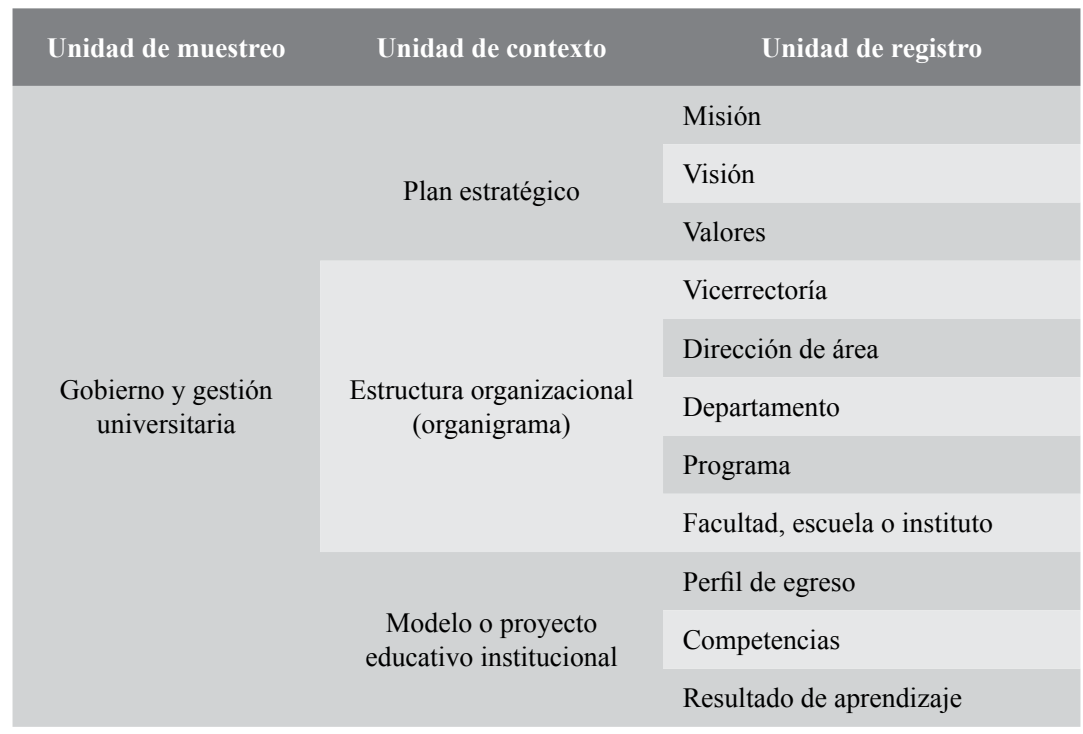

Fuente: elaboración propia con base en Palomares et al., 2012. 
Así, en esta investigación se analiza en los planes estratégicos la aparición o no del concepto de responsabilidad social en la declaración de misión, visión y valores institucionales, así como su existencia concreta en un área dentro del organigrama institucional y, su mención dentro de los modelos educativos de cada universidad en cuanto al perfil de egreso, competencias o resultados de aprendizaje.

\section{Resultados}

\section{Planes estratégicos.}

En términos generales, como se observa en la gráfica 1 , el $44 \%$ de los planes estratégicos en estudio (siete universidades) no contempla ningún tipo de uso de la locución RSU como un discurso institucional de carácter es- tratégico, ya sea en la misión, visión o valores institucionales. Al contrario, nueve universidades estatales (56\%) utilizan esta expresión en uno o más de los tres elementos de su plan estratégico.

\section{Gráfica 1}

Responsabilidad social universitaria en los planes estratégicos de las universidades estatales chilenas

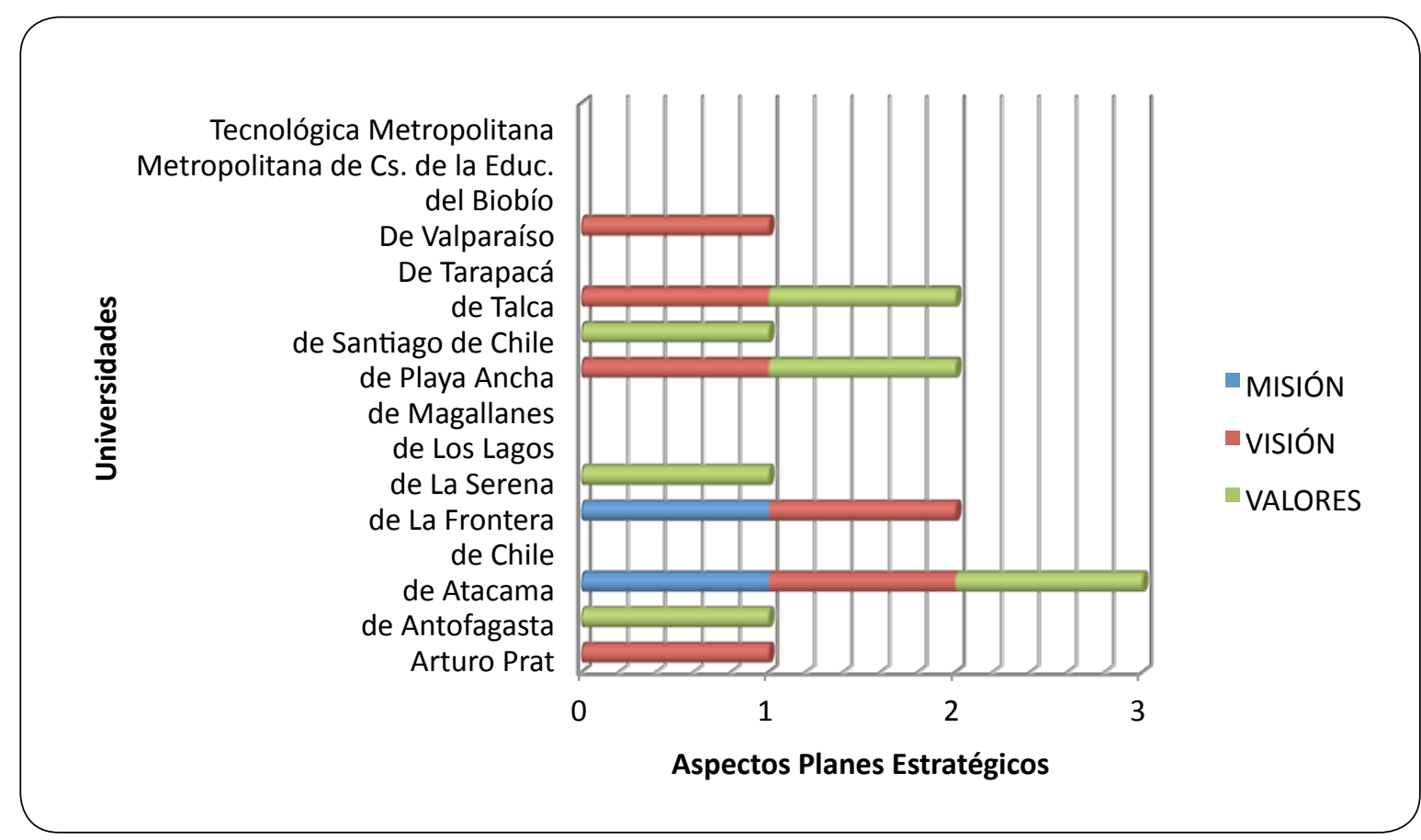

Fuente: elaboración propia.

En cuanto a las universidades estatales que sí consideran a la RSU en algunos de los aspectos de los planes estratégicos en estudio, cuatro pertenecen a la macrozona norte (Universidad Arturo Prat, Universidad de Antofagasta,
Universidad de Atacama y Universidad de La Serena), dos a la zona sur (Universidad de Talca y Universidad de La Frontera) y tres a la zona central (Universidad de Valparaíso, Universidad de Playa Ancha y Universidad de Santiago 
de Chile), observándose una distribución por zona geográfica bastante equilibrada.

Así mismo, la gráfica 1 evidencia que con excepción de la Universidad de Santiago de Chile, únicamente las universidades regionales contemplan el uso del término RSU en alguno de los tres aspectos analizados en los planes estratégicos. Además, la Universidad de Atacama es la única institución de la muestra que emplea dicho término simultáneamente en la misión, visión y valores institucionales.

Estos resultados nos permiten observar las diferencias existentes entre las universidades estatales de carácter regional y las metropolitanas, en cuanto a la presencia del término RSU en este primer elemento del gobierno y la gestión universitaria, lo cual es importante porque el plan estratégico institucional es la principal herramienta que usan las organizaciones para orientar la toma de decisiones y el establecimiento de las prioridades dentro del presupuesto institucional, por lo que si la RSU no está declarada en esta instancia no logra retroalimentar realmente los procesos decisionales de las universidades.

\section{Misión institucional.}

Para realizar una comparación más homogénea entre estos documentos, se analizaron los textos de la misión, visión y valores institucionales, para determinar el grado de presencia de la RSU en cada uno de ellos, resultados que se muestran en la gráfica 2 .

Gráfica 2

Responsabilidad social universitaria en los planes estratégicos de las universidades estatales chilenas

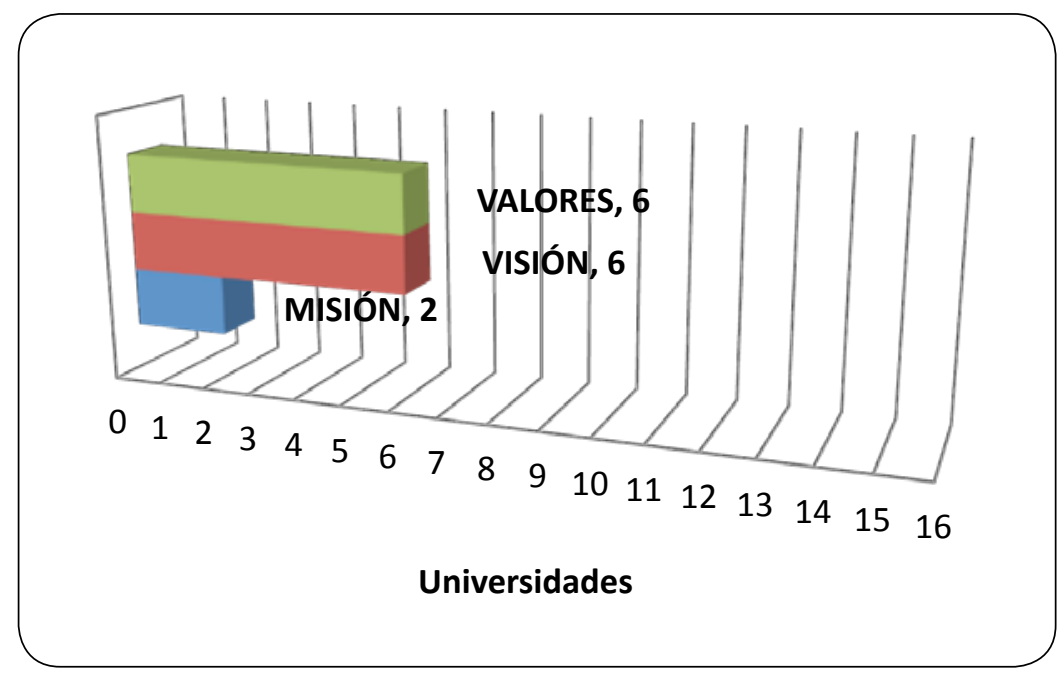

Fuente: elaboración propia.

Como se observa en la gráfica 2, la presencia de la RSU como un aspecto relevante dentro de los planes estratégicos de las universidades estatales chilenas es más bien bajo, especialmente en la declaración de misión institucional en donde tan solo dos universidades de la muestra incorporan la expresión "socialmente responsable" dentro del texto de este elemento estratégico.

Los extractos de las misiones de ambas instituciones ya expuestos, aunque reportan la escasa participación de la RSU en este aspecto de los planes estratégicos que expresa o declara 
la razón de ser de una organización, sí nos muestran uno de los ámbitos habituales de aplicación de este modelo en las universidades: la formación.

Sin embargo, la baja consideración de la RSU como un factor de la redacción en la misión institucional, puede tomarse como un indicador de una limitada aplicación del comportamiento socialmente responsable en el quehacer institucional, dado que si bien se trata de una declaración dentro del plan estratégico, puede ser reflejo de la manera en la cual se desarrollan las actividades, estrategias y políticas institucionales para conseguir sus objetivos y metas.

Además, si se tiene en cuenta que por lo regular las instituciones utilizan los textos de su declaración de misión en diferentes documentos oficiales, página web o inclusive en las estrategias de difusión o marketing, se está desperdiciando un espacio cardinal para difundir la responsabilidad social, en cuanto estas declaraciones normalmente son aspectos bastante reconocidos por la comunidad universitaria y pueden ser una instancia valiosa para introducir a la RSU en la cultura organizacional, así como para sensibilizar a los docentes, estudiantes y funcionarios sobre la importancia del concepto de RSU, situación que lamentablemente en el caso estudiado no es aplicable.

\section{Visión institucional.}

En lo que respecta a la declaración de visión, existe un leve aumento en el número de universidades que incluyen algún tipo de referencia a la RSU en este nivel, donde seis universidades (Arturo Prat, de Atacama, de La Frontera, de Playa Ancha, de Talca y de Valparaíso) muestran en su visión elementos que aluden a este aspecto, destacando el hecho de que dos universidades que consideran en su misión a la RSU también lo hacen en este segundo aspecto analizado en los planes estratégicos.
Los resultados alcanzados en los dos primeros aspectos de la planificación estratégica en estudio, plantean que exclusivamente las universidades regionales incorporan a la RSU en las declaraciones de misión y visión, donde solo la Universidad de Atacama y la Universidad de La Frontera registran en ambos elementos referencias a la RSU.

Ambas universidades muestran perfiles institucionales disímiles según lo señalado en la tabla 2, ya que la Universidad de Atacama tiene cuatro años de acreditación y la Universidad de La Frontera cinco años; la segunda cuenta con más del doble de alumnos matriculados durante el año 2015 que la primera, y solamente en cuanto al establecimiento de origen de sus estudiantes existe cierta similitud, en los dos casos provienen en un $97 \%$ de instituciones municipales o subvencionadas.

De esta manera, la mayoría de universidades en examen no evidencia la presencia de la responsabilidad social en la declaración de visión institucional, mientras que su incorporación podría considerarse como la expresión de la aspiración de la universidad por alcanzar un comportamiento socialmente más responsable en el mediano y largo plazo, debido a que este aspecto de un plan estratégico describe lo que una institución espera alcanzar o transformarse en el futuro.

\section{Valores institucionales.}

Por último, la revisión de los planes estratégicos de las universidades estatales chilenas consideró a los valores y principios institucionales declarados en dichos documentos, registrándose un resultado similar en este elemento que lo obtenido para el caso de la visión institucional, donde seis universidades declaran a la responsabilidad social como un valor institucional, como se evidenció en la gráfica 2.

No obstante lo anterior, al revisar las definiciones que cada una de las seis universidades 
plantea para la responsabilidad social como valor institucional, se observan algunos ejes relevan- tes que buscan hacer más operativo este aspecto deontológico (valores) (véase gráfica 3 ).

Gráfica 3

Aspectos relevantes en la definición de la responsabilidad social universitaria como valor institucional

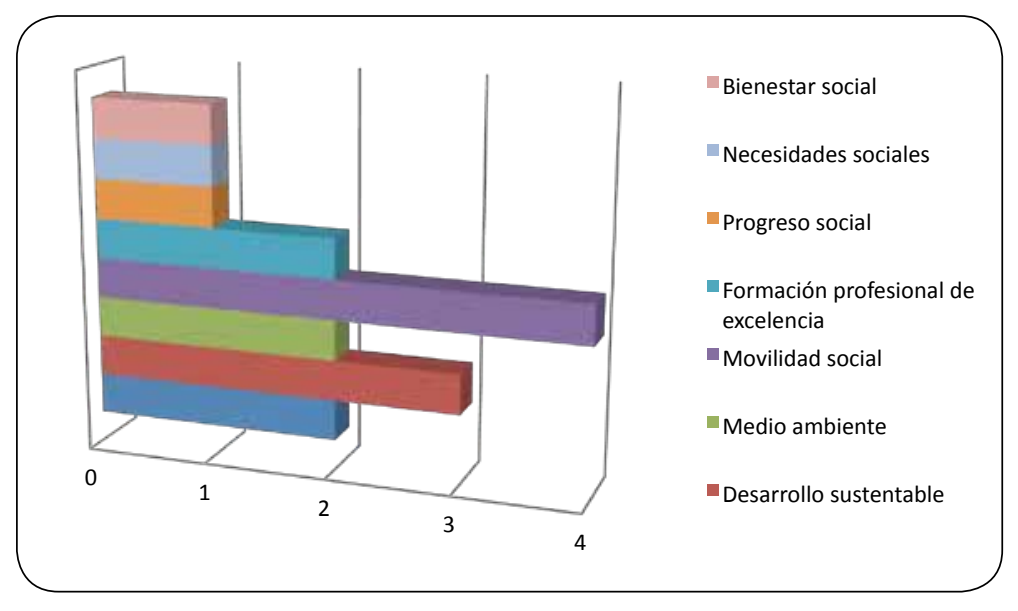

Fuente: elaboración propia.

En la gráfica 3 se muestra que la movilidad social es la perspectiva que registra la mayor frecuencia cuando se analiza a la responsabilidad social como un valor institucional (Universidad de Antofagasta, Universidad de Atacama, Universidad de La Serena, Universidad de Playa Ancha, Universidad de Santiago de Chile y Universidad de Talca). Para tales instituciones de educación superior, dicho aspecto se transforma en un elemento cardinal de su comportamiento socialmente responsable, por lo que han decidido plasmarlo en sus planes estratégicos como un valor institucional de gran importancia, lo que repercute sobre todo en la cultura organizacional de este tipo de universidades.

Los resultados obtenidos indican que solo el $56 \%$ de las universidades estatales chilenas utiliza el término responsabilidad social como un aspecto significativo en su misión, visión o valores institucionales. La Universidad de Atacama es la única institución dentro de la muestra que considera dicho concepto en los tres elementos estudiados en los planes estratégicos (misión, visión y valores).
Sin embargo, aunque con una frecuencia baja, resulta interesante observar que la movilidad social está presente en las declaraciones de misión y de valores institucionales, siendo susceptible de vincular con el comportamiento socialmente responsable de las universidades estatales. Lo anterior, habla de la relación con los procesos de masificación de la educación superior desarrollados en Chile que han permitido a un gran número de jóvenes acceder a la universidad, teniendo la oportunidad de mejorar su situación socioeconómica a través del acceso a empleos con una remuneración mayor, si se los compara con aquellos jóvenes con educación secundaria que ingresan directamente al mercado laboral.

\section{Estructura organizacional (organigramas).}

En este segundo elemento (variable para examinar la aparición de la responsabilidad social en el gobierno y gestión de las universidades estatales chilenas), se analizan los organigramas de cada institución para identificar si existen unidades o áreas dentro de su estructura organizacional encargadas de im- 
plementar las acciones que se enlazan con la $\mathrm{RSU}$, para reconocer tanto el nivel jerárquico en el cual está ubicada como el área de dependencia (véase tabla 4).

Tabla 4

La responsabilidad social universitaria en la estructura organizacional de las universidades estatales chilenas

\begin{tabular}{|c|c|}
\hline Universidad & Denominación y dependencia jerárquica \\
\hline de Chile & Unidad Nexo RSU dependiente de la Facultad de Economía y Negocios \\
\hline de La Frontera & $\begin{array}{l}\text { Unidad de Responsabilidad Social dependiente de la Dirección Académica de Pregrado de la Vi- } \\
\text { cerrectoría Académica }\end{array}$ \\
\hline de Los Lagos & $\begin{array}{l}\text { Dirección de Vinculación con el Medio y Responsabilidad Social dependiente de la Vicerrectoría } \\
\text { Académica }\end{array}$ \\
\hline de Santiago de Chile & $\begin{array}{l}\text { Programa de RSU dependiente del Departamento de Extensión de la Vicerrectoría de Vinculación } \\
\text { con el Medio }\end{array}$ \\
\hline de Talca & $\begin{array}{l}\text { Dirección Corporativa de Responsabilidad Social Universitaria dependiente de la Rectoría de la } \\
\text { Universidad }\end{array}$ \\
\hline del Bío-Bío & $\begin{array}{l}\text { Programa de Responsabilidad Social dependiente de la Dirección de Desarrollo Estudiantil de la } \\
\text { Vicerrectoría Académica }\end{array}$ \\
\hline
\end{tabular}

Fuente: elaboración propia.

La tabla 4 nos muestra que tan solo el 38 $\%$ de las universidades estatales chilenas en estudio posee una unidad administrativa o académica encargada de la RSU dentro de su estructura organizacional. No obstante, tres de las universidades incluidas en dicha tabla (de Chile, de Los Lagos y del Bío-Bío), no tienen ninguna utilización del término responsabilidad social en los aspectos analizados de los planes estratégicos (visión, misión y valores), situación que podría tomarse como una incoherencia dentro del gobierno y gestión de las mismas, ya que no es relevante dentro del principal instrumento orientador para la toma de decisiones institucionales como lo es el plan estratégico, pero sí se encuentra reflejada en sus organigramas.

Otro aspecto significativo es que tres de las unidades encargadas de la RSU expuestas en la tabla 4 (de La Frontera, de Los Lagos y del Bío-Bío) pertenecen a una dirección dependien- te de la vicerrectoría académica. Sin embargo, dichas direcciones encargadas de la RSU en estas universidades son bastante diferentes entre sí: Dirección Académica de Pregrado, Vinculación con el Medio, Desarrollo Estudiantil.

Lo anterior, nos permite decir que si bien dependen de una misma vicerrectoría, las orientaciones más directas de las acciones de RSU que pudieran emprenderse pueden ser más internas en los casos de la Universidad de $\mathrm{La}$ Frontera y la Universidad del Bío-Bío, y más hacia el exterior en el caso de Vinculación con el Medio en la Universidad de Los Lagos.

Luego, en el caso de la Universidad de Chile, la existencia de la Unidad Nexo RSU dentro de la Facultad de Economía y Negocios plantea un ámbito organizacional distinto, ubicando a la responsabilidad social en una instancia más 
académica que administrativa, a diferencia del resto de los casos identificados en la muestra.

Ahora bien, la Universidad de Talca tiene condiciones especiales ya que se trata de una dirección corporativa dependiente directamente del rector de la institución, rompiendo con ello la tendencia más tradicional del resto de los casos identificados que ubican a la RSU dentro de una unidad administrativa dependiente de la vicerrectoría académica, o de la Vicerrectoría de Vinculación con el Medio como en el caso de la Universidad de Santiago de Chile.

\section{Modelos educativos.}

Una de las manifestaciones habituales de la RSU se relaciona con la formación de los profesionales universitarios orientada a que los estudiantes adquieran un mayor compromiso social, ético y ciudadano en el ejercicio de su profesión, razón por la cual, la universidad socialmente responsable debe realizar esfuerzos y destinar recursos para alcanzar dichos propósitos incorporando este elemento a los modelos educativos.

En este sentido, analizamos los modelos o proyectos educativos de las universidades estatales chilenas, específicamente en cuanto a los perfiles de egreso, competencias o resultados de aprendizaje por competencia, para verificar si se considera a la responsabilidad social como un factor dentro de esta importante instancia estratégica en el desarrollo de la docencia universitaria.

\section{Gráfica 4}

La responsabilidad social universitaria en los modelos educativos de las universidades estatales chilenas

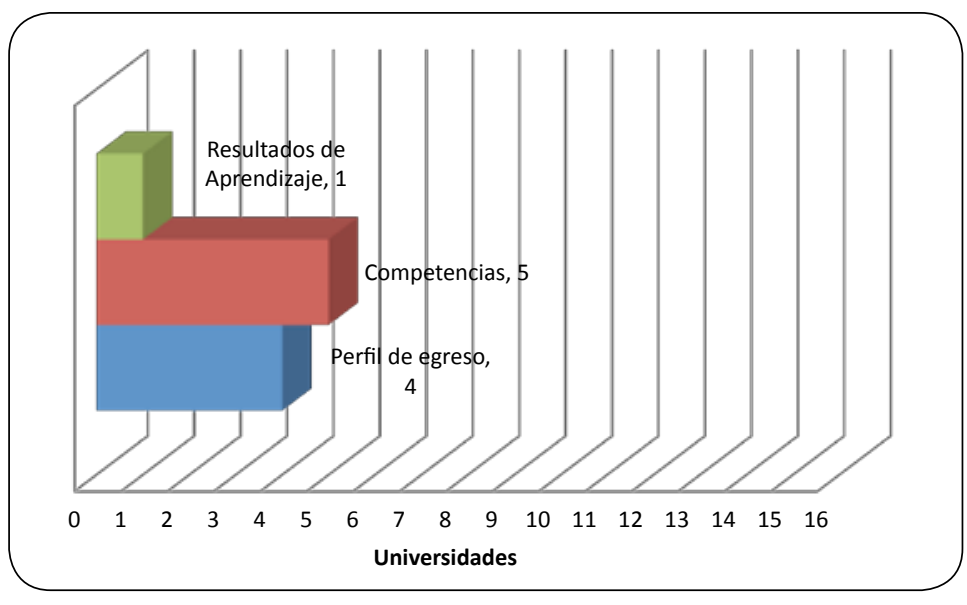

Fuente: elaboración propia.

La existencia de la responsabilidad social en los modelos educativos es más destacada en las competencias y perfiles de egreso, aunque en ambos casos no superan el $31 \%$ y $25 \%$ respectivamente, mientras que en los resultados de aprendizaje es casi inexistente, siendo este último aspecto de carácter más operativo dentro de cada modelo educativo y permitiría plasmar en cuestiones mucho más concretas, el desarrollo de la responsabilidad social dentro de la formación de los profesionales universitarios.

Al respecto, la gráfica 5 reporta la distribución de universidades que registran a la responsabilidad social en alguno de los elementos de los modelos educativos analizados, donde su consideración como competencia alcanza la mayor frecuencia. 
Gráfica 5

Presencia de la responsabilidad social universitaria

en los modelos educativos, según universidad

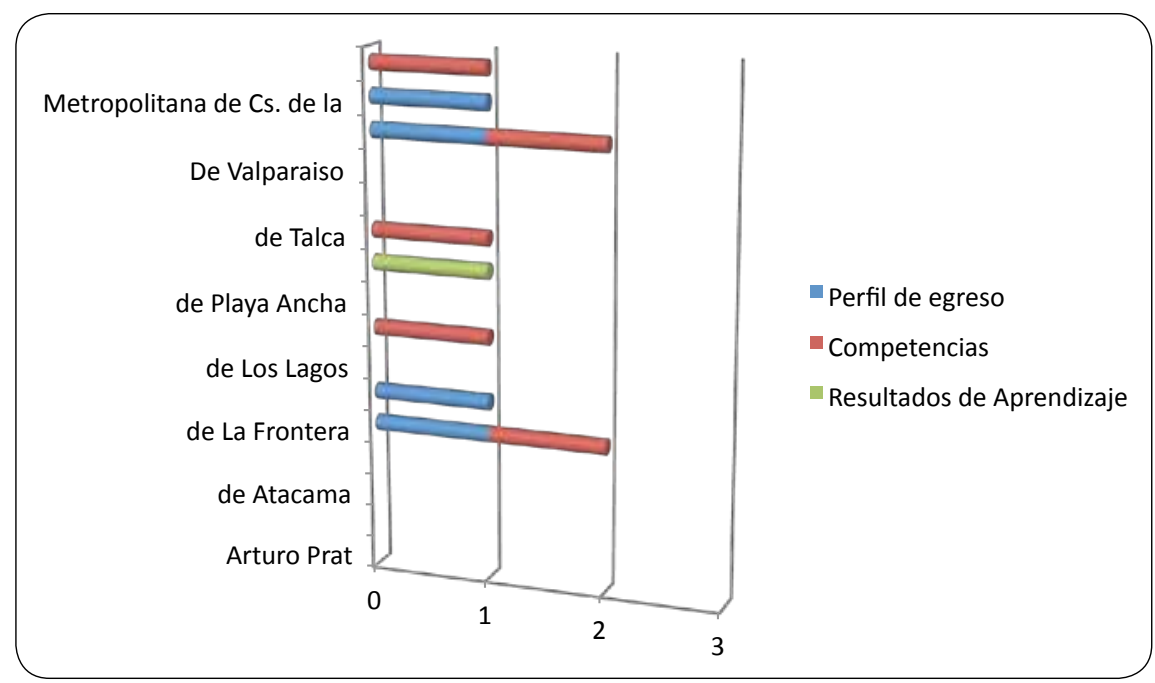

Fuente: elaboración propia.

Ahora bien, como se observa en la gráfica 5, la Universidad de La Frontera y la Universidad del Bío-Bío consideran a la responsabilidad social tanto en el perfil de egreso como también una competencia, transformándose en los casos más destacados en este ámbito. Por último, en el caso de los resultados de aprendizaje por competencia, únicamente la Universidad de Santiago de Chile valora a la responsabilidad social en esta categoría.

Estos resultados nuevamente muestran una presencia parcial y más bien baja de la RSU dentro de los modelos educativos de las universidades estatales chilenas, existiendo una dispersión importante de instituciones que consideran al comportamiento socialmente responsable en esta instancia, y como fuera señalado anteriormente, solo en los casos de la Universidad de La Frontera y la Universidad del Bío-Bío se coincide en incorporarlos tanto en el perfil de egreso como en las competencias de formación.

\section{Análisis de los resultados.}

De acuerdo con los resultados expuestos aquí, la responsabilidad social es un aspecto que está presente solo parcialmente en algunas universidades estatales chilenas, siendo sin duda alguna el caso más sobresaliente el de la Universidad de La Frontera, institución que incorpora este factor en todos los elementos de gobierno y gestión universitaria analizados, es decir, en su misión y visión y también como unidad administrativa dentro de su estructura organizacional.

El único componente en el que la Universidad de La Frontera no tiene en cuenta a la responsabilidad social es el de valores institucionales, no obstante es importante señalar que esta institución asume todos los valores y principios de la universidad socialmente responsable del proyecto UCP, lo cual compensa en absoluto este aspecto y nos revela a una universidad modélica dentro de la muestra en estudio. Lo precedente se complementa con el hecho de que esta universidad también integra el proyecto MECESUP que busca desarrollar un modelo de enseñanza de la responsabilidad social.

Otras variables que pueden resultar útiles para analizar la RSU es el origen de los alumnos matriculados en cada una de las universidades 
estatales chilenas en examen. Considerando aquellas instituciones que matriculan el $40 \%$ o más de sus estudiantes de primer año con esta característica, observamos en la tabla 5 la baja presencia de universidades que declaran a la RSU como parte de su gobierno y gestión universitaria.

Tabla 5

Responsabilidad social universitaria y universidades estatales con mayor porcentaje de alumnos de liceos municipales

\begin{tabular}{lccccccc} 
& $\begin{array}{c}\text { \% alumnos liceos } \\
\text { municipales }\end{array}$ & Visión & Misión & Valores & Organigrama & $\begin{array}{c}\text { Perfil de } \\
\text { egreso }\end{array}$ & Competencias \\
\hline de Los Lagos & 53,9 & No & No & No & Sí & No & No \\
de Magallanes & 40,8 & No & No & No & No & No & Sí \\
de Talca & 40,2 & Sí & No & Sí & Sí & No & Sí
\end{tabular}

Fuente: elaboración propia.

Según la tabla 5, entre las instituciones con alta matrícula de origen en los liceos municipales, únicamente la Universidad de Talca considera a la responsabilidad social en los tres aspectos del gobierno y gestión universitaria, mientras que la Universidad de Tarapacá no la incluye en ninguno. Además, la Universidad de Los Lagos posee el mayor porcentaje de alumnos provenientes de liceos municipales matriculados en 2015, aunque solo asume la responsabilidad social en uno de los aspectos en análisis. Es un caso relevante porque cuenta con una Dirección de Vinculación con el Medio y Responsabilidad Social, lo cual sin duda le permitirá a esta institución desarrollar esfuerzos significativos en un ámbito relevante para la RSU como lo es la relación de la universidad con la sociedad.

Estos resultados demuestran que la RSU no es un aspecto especialmente presente en los planes estratégicos, organigramas y modelos educativos de las universidades estatales chilenas que matriculan un mayor porcentaje de alumnos provenientes de establecimientos municipales, con las excepciones antes mencionadas.

Otra cuestión interesante se relaciona con estudiar la mayor presencia de la responsabilidad social en el gobierno y la gestión universitaria entre aquellas instituciones con más tiempo de acreditación, situación que se puede observar en la tabla 6 .

Tabla 6

Responsabilidad social universitaria y universidades estatales con más años de acreditación institucional

\begin{tabular}{|llcccccc} 
& Años de acreditación & Visión & Misión & Valores & Organigrama & $\begin{array}{c}\text { Perfil de } \\
\text { egreso }\end{array}$ & Competencias \\
\hline de Chile & Siete años & No & No & No & Sí & No & No \\
\hline de Santiago de Chile & Seis años & No & No & Sí & Sí & No & No \\
\hline de La Frontera & Cinco años & Sí & Sí & No & Sí & Sí & Sí \\
\hline de Talca & Cinco años & Sí & No & Sí & Sí & No & Sí \\
\hline de Tarapacá & Cinco años & No & No & No & No & No & No \\
\hline de Valparaíso & Cinco años & Sí & No & No & No & No & No \\
del Bío-Bío & Cinco años & No & No & No & Sí & Sí & Sí \\
\hline
\end{tabular}

Fuente: elaboración propia. 
De acuerdo con la tabla 6, el único escenario donde se puede observar una relación importante entre las universidades con varios años de acreditación y la presencia de la RSU es en la estructura organizacional, debido a que cinco universidades de este subgrupo han desarrollado alguna unidad administrativa o académica encargada de las acciones y avances en la aplicación de la responsabilidad social en su quehacer. Luego, en el ámbito individual, la Universidad de La Frontera, la Universidad de Talca y la Universidad del Bío-Bío son las que presentan un mayor despliegue de la RSU en los tres aspectos de gobierno y gestión universitaria analizados.

\section{Conclusiones}

La presencia del concepto de responsabilidad social en las declaraciones y planteamientos de las universidades estatales chilenas en estudio es más bien bajo, lo que en la práctica se traduce en que los rectores, directivos universitarios y en general, las comunidades universitarias de las instituciones analizadas, no incorporen este elemento a su trabajo cotidiano.

Relacionado con lo anterior, resulta llamativa la baja aparición del término RSU en los textos de las declaraciones de misión institucional de las universidades de la muestra, porque es en este nivel del plan estratégico donde se define la razón de ser o quehacer institucional; es decir, donde se establece el qué se hace o aquello a lo que se dedica.

Además, la baja presencia de la RSU en los planes estratégicos también se refleja en la estructura organizacional de las universidades estatales chilenas, donde tampoco cuentan con muchas formas de expresión a través de unidades administrativas o académicas, encargadas de implementar acciones que se vinculan con el comportamiento socialmente responsable de la universidad.

Una situación similar se observa para lo referido a los modelos educativos, instancia en donde la responsabilidad social se evidencia mayormente como competencia genérica y en algunos perfiles de egreso, lo que incluye la innovadora propuesta de cuatro universidades chilenas que actualmente están implementando un modelo para la enseñanza de la responsabilidad social, donde participan la Universidad de La Frontera y la Universidad de Talca que formaron parte de la muestra de este estudio.

Lo anterior, no implica necesariamente un actuar socialmente irresponsable de las universidades de la muestra, pero sí puede transformarse en una barrera para avanzar de forma más rápida y contundente en la incorporación de la RSU a la cultura y quehacer institucional en el largo plazo, como un elemento de la política pública de educación superior en Chile, tal como ocurre con el acceso y la calidad.

En ese sentido, cuando se examina el tipo de estudiantes que acceden a las universidades estatales chilenas, aquellas instituciones que matriculan una mayor cantidad de alumnos provenientes de establecimientos municipales, es decir, con una mayor vulnerabilidad social, no contemplan a la RSU en las instancias de gobierno y gestión universitaria analizada. Esa circunstancia podría ser un indicador de que las mayores tasas de matrícula de alumnos más vulnerables se deberían sobre todo a los efectos de la propia oferta y demanda del mercado universitario chileno, y no necesariamente a una política institucional que privilegie la matrícula de ese tipo de estudiantes, buscando una más alta RSU.

Luego, al considerar la relación entre la presencia de la RSU en el gobierno y gestión universitaria y la calidad, expresada en los años de acreditación institucional de cada universidad, aquellas instituciones de la muestra con mayor nivel de acreditación sí poseen en sus organigramas institucionales una unidad encargada de coordinar a la RSU, lo que podría entenderse como una asociación entre calidad y comportamiento socialmente responsable en las universi- 
dades. Pero además, abre una oportunidad interesante para que dichas universidades incorporen los procedimientos y estándares de calidad en el funcionamiento de las unidades responsables de la RSU, para que las acciones desarrolladas por dichas instancias garanticen también un nivel de calidad significativo de forma que sus impactos puedan perdurar en el largo plazo.

Así, resulta fundamental que las universidades que quieran fortalecer su comportamiento socialmente responsable, integren a la RSU en las instancias más cardinales del gobierno y gestión universitaria. Ello posibilitará retroalimentar las decisiones estratégicas que se tomen en el interior de cada universidad con los criterios y elementos propios del concepto de RSU, permitiendo a las universidades mejorar o aumentar su contribución efectiva al desarrollo de la sociedad desde su quehacer de docencia e investigación, lo que en el caso chileno facilita llegar a expresarse además en una conexión más fuerte entre la RSU y el acceso y la calidad de las universidades, articulándose dicha interacción en los planes estratégicos, organigramas y modelos educativos que cada universidad desarrolla.

\section{Nota}

${ }^{1}$ Prueba de Selección Universitaria.

\section{Referencias}

Arango, O., Clavijo, S., Puerta, I., \& Sánchez, J. (2014). Formación académica, valores, empatía y comportamientos socialmente responsables en estudiantes universitarios. Revista de la Educación Superior, 43(169), 89-105.

Aristimuño, M., \& Rodríguez, C. (2014). Responsabilidad social universitaria. Su gestión desde la perspectiva de directivos y docentes. Estudio de caso: una pequeña universidad latinoamericana. Interciencia, 39(6), 375-382.
Asociación de Universidades confiadas a la Compañía de Jesús en América Latina -[AUSJAL]. (2009). Presentación sintetizada del sistema de autoevaluación y gestión de la responsabilidad social universitaria en AUSJAL. Argentina: Autor. Recuperado de http://www.ausjal.org/ tl_files/ausjal/images/contenido/Documentos/Publicaciones/Documentos $\% 20$ Institucionales/RSU\%20AUSJAL\%20 version $\% 20$ acotada.pdf

Atria, R. (2006). La gestión de las universidades del Consejo de Rectores. Revista Calidad en la Educación, 24, 65-116.

Brunner, J. (2011). Gobernanza universitaria: tipología, dinámicas y tendencias. Revista de Educación, 355, 137-159.

Castro, D. y Tomás, M. (2010a). El desempeño de la dirección en la universidad: el caso de Decanos y Directores de Departamento. Revista Educación XX1, 13(2), 217-239.

Castro, D., \& Tomás, M. (2010b). El gobierno y la gestión de la universidad: estudio de los órganos unipersonales. Estudios sobre Educación, 19, 165-184.

Comisión Europea (2008). Higher Education Governance in Europe. Policies, structures, funding and academic staff. Bruselas: Eurydice.

Fundación Universitaria Luis Amigó. (2015). Experiencias iberoamericanas en responsabilidad social universitaria. Medellín: Funlam. Recuperado de http://www. funlam.edu.co/uploads/fondoeditorial/ ebook/2015/books gratis-Experienciasiberoamericanas-en-responsabilidad.pdf

Gaete, R. (2015). El concepto de responsabilidad social universitaria desde la perspectiva de la alta dirección. Revista Cuadernos de Administración, 31(53), 97-107. 
Gaete, R. (2012). Gobierno universitario pluralista. Una propuesta de análisis desde la teoría de los stakeholders. Revista de Universidad y Sociedad del Conocimiento, 9(2), 115-129. Recuperado de http://www.raco.cat/index.php/RUSC/ article/viewFile/284628/372855

Gaete, R. (2011). La responsabilidad social universitaria como desafío para la gestión estratégica de la educación superior: el caso de España. Revista de Educación, 355, 109-133.

Ganga, F. (2005). Análisis preliminar del gobierno universitario chileno. Revista Venezolana de Gerencia, 10(30), 213-246.

Jiménez, M. (2002). La Universidad Construye País. Santiago de Chile: Corporación Participa. Recuperado de http:// rsuniversitaria.org/web/images/stories/ memoria/UCP\%202002.pdf

Jongbloed, B., \& Goedegebuure, L. (2003). De la universidad emprendedora a la universidad stakeholder. En J. Vilalta \& E. Pallejá (Eds.), Universidades y desarrollo territorial en la sociedad del conocimiento (pp. 153-178). Barcelona: Universidad Politécnica de Cataluña.

Larrán, M., \& Andrades, F. (2015). Análisis de la responsabilidad social universitaria desde diferentes enfoques teóricos. $R e$ - vista Iberoamericana de Educación Superior, 6(15), 91-107.

Lopera, C. (2004). Antinomias, dilemas y falsas premisas que condicionan la gestión universitaria. Revista Mexicana de Investigación Educativa, 9(22), 617-635.

Meléndez, M.; Solís, P. \& Gómez, J. (2010). Gobernanza y gestión de la universidad pública. Revista de Ciencias Sociales, 16(2), 210-225.

Ojeda, J. (2013). Responsabilidad social, construcción de un concepto desde la percepción del docente universitario. Revista Teorías, enfoques y aplicaciones en las Ciencias Sociales (TEACs), 5(12), 11-24.

Palomares, D., García, A., \& Castro, E. (2012). Misiones actuales de las universidades públicas: una perspectiva sociológica. Revista Arbor, 188(753), 171-192.

Rodríguez, E. (2006). El proceso de toma de decisiones estratégicas en las universidades públicas. Revista Calidad en la Educación, 24, 47-63.

Vallaeys, F. (2008). Responsabilidad Social Universitaria: una nueva filosofía de gestión ética e inteligente para las universidades. Revista Educación Superior y Sociedad, 13(2), 191-220. 